\title{
Wrong organic crystal structures solved and refined from powder data
}

\section{Martin U. Schmidt, Viktoria Gramakov, Carina Schlesinger, Lukas Tapmeyer}

\author{
Goethe-Universität, Institut für Anorganische und Analytische Chemie, Max-von-Laue-Str. 7, 60438 Frankfurt am Main, Germany. \\ m.schmidt@chemie.uni-frankfurt.de
}

Organic crystal structures, solved and refined from powder data, may be fully wrong, even if they are chemically sensible and give a good fit to the powder patterns. Two examples are shown.

In both cases, the atomic positions and the molecular packing were completely wrong.

Example 1:

The crystal structure of the commercial organic hydrazone Pigment Red 52:1, $\mathrm{Ca}^{2+}\left(\mathrm{C}_{18} \mathrm{H}_{11} \mathrm{ClN}_{2} \mathrm{O}_{6} \mathrm{~S}\right)^{2-*} \mathrm{H}_{2} \mathrm{O}$ was determined from powder data in the usual way by indexing, structure solution by real-space methods, and Rietveld refinement. The resulting structure was chemically sensible and gave a good fit to the powder data. By chance, a single-crystal of poor quality was obtained, and the correct structure was determined by a combination of single-crystal structure analysis and Rietveld refinement. The structure initially determined from powder data turned out to be completely wrong. The correct and the wrong structures differ in the position and coordination of the $\mathrm{Ca}^{2+}$ ions, as well as the position and mutual arrangement of the anions (see Figure).

Example 2:

The crystal structure of 4,11-difluoroquinacridone, $\mathrm{C}_{20} \mathrm{H}_{10} \mathrm{~F}_{2} \mathrm{~N}_{2} \mathrm{O}_{2}$, was solved from unindexed powder data by a global fit of millions of random structures to the powder pattern using the FIDEL method [1,2], which uses cross-correlation functions for the comparison of experimental and simulated powder patterns. The structures were subsequently refined by the Rietveld method. Four completely different structures (different space groups, different molecular packings, different $\mathrm{H}$ bond topolgies) were obtained. All four structures were chemically sensible, had a good fit to the powder data, gave a relibale fit to the pair-distribution function and good lattice energy minimisation. One is correct, the other were wrong [3].

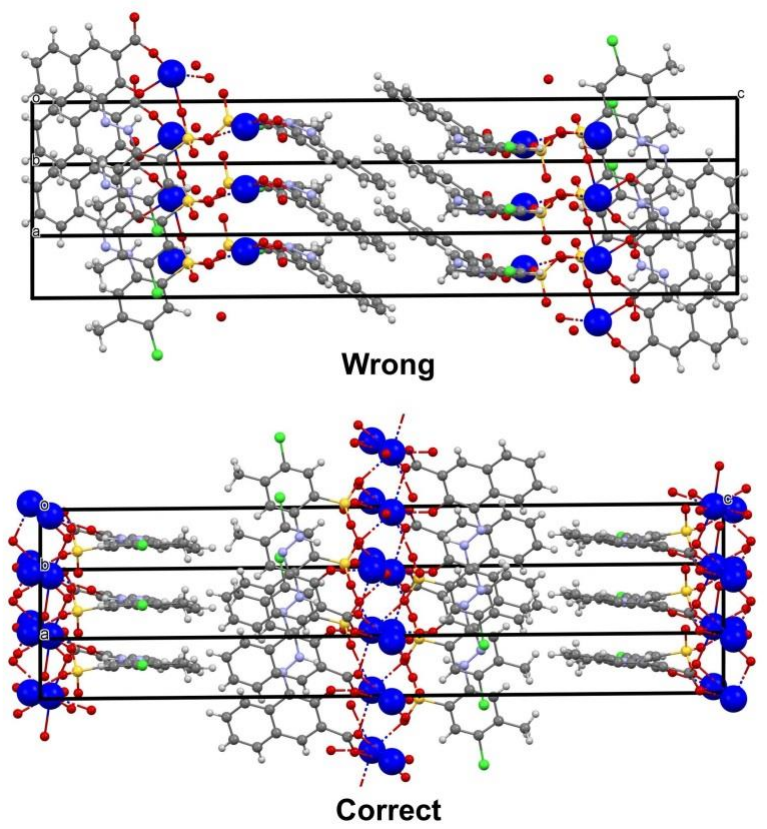

[1] S. Habermehl, P. Mörschel, P. Eisenbrandt, S.M. Hammer, M.U. Schmidt, Acta Cryst. B70 (2014), $347-359$.

[2] S. Habermehl, C. Schlesinger, M.U. Schmidt, in preparation.

[3] C. Schlesinger, A. Fitterer, C. Buchsbaum, S. Habermehl, M.U. Schmidt, in preparation.

Keywords: Structure determination from powder data, organic compounds, wrong crystal structures, Rietveld refinement, pair-distribution function refinement 